\title{
DWT-PSD EXTRACTION FEATURE FOR DEFECT DIAGNOSIS OF SMALL WIND GENERATOR
}

\author{
Lahcène NOUREDDINE, Mustapha NOUREDDINE, Ahmed HAFAIFA, Abdellah KOUZOU
}

\author{
Applied Automation and Industrial Diagnostic Laboratory, Ziane Achour University of Djelfa, \\ nourlahc@gmail.com
}

\begin{abstract}
In this paper, the ability to detect broken rotor bar (BRB) defects in a small renewable energy system (based on a squirrel cage induction generator (SCIG)) by the digital signal processing of captured phase currents, is presented. The new approach proposed in this study is a combination of two techniques. The first technique is a discrete wavelet transform (DWT) by the decomposition of the phase current signal in multilevel frequency bands. This is performed with the analysis of some selected approximations and/or details, which contain both the lower and upper sideband components presenting the characteristic frequency of the BRB fault. The second technique is power spectral density (PSD) analysis. This approach provides the ability to optimize the diagnosis of rotor defects in electrical generators. The results obtained by the proposed DWT-PSD approach are proved and improved by comparing them with the results of the PSD analysis, obtained from the original phase current signal delivered by the $5.7-\mathrm{kW}$ squirrel cage induction generator, based on a small wind energy conversion system.
\end{abstract}

Keywords: discrete wavelet transform; BRB defects diagnosis; PSD estimation; renewable energy system.

\section{INTRODUCTION}

Digital signal processing (DSP) techniques applied in the field of fault diagnosis have exhibited tremendous progress over the past few decades $[1$, $2]$. The gradual implementation of approved and improved DSP techniques has been extremely useful for the diagnosis of electrical machines, mainly aimed at evolving modern techniques to diagnose possible faults. Provided these faults are in the first stages, large and expensive damage can be avoided. This permits industrial users to take appropriate maintenance actions early, to reduce the potential costs caused by unexpected stops. The use of DSP techniques allows maximum reliability and precision for making necessary decisions related to a device state; therefore, it is possible to reach additional facts once these techniques are implemented.

Phase current analysis has attracted notable attention, because phase current signals can be captured easily, and it is commonly used in tools for sensing and analysis [3]. In addition, phase currents or other signatures (fluxes, vibrations [4, 5], and voltages) are frequently non-stationary, which makes the use of traditional signal treatment methods complex (or even unattainable) (e.g., Fourier transform (FT)). For the analysis of such signals, and for obtaining maximum information, modern DSP methods, which are convenient for analyzing unsteady signatures, should be employed.

Therefore, among the advanced wavelet transform (WT) methods, an empirical WT (EWT) is employed for the generator rolling bearing and gearbox fault diagnosis for wind turbines by using measured vibration signals [6-9]. The integration of resonance-based sparse signal decomposition (RSSD) and wavelet transform is used to detect rolling bearing faults [10]. Other advanced methods employed for a similar diagnosis include rational dilation WT (RDWT), tunable Q-factor WT (TQWT), and Q-factor Gabor wavelets (QGW) [11-13]. Indeed, each of the above methods is capable of following the developments of the several frequencies generated by the characteristic mechanical defects caused by the processed signatures (vibration or speed signals). Diagnosis of frequency development is a reliable indicator of the existence of failure, because the generation of these frequencies cannot be reasoned by another phenomenon.

The combination of a discrete WT (DWT) and the power spectral density (PSD) is an important contribution to the broken rotor bar (BRB) fault diagnosis of a small wind generator by using measured phase current signals. Until recently, this was not studied. Therefore, the use of electrical signatures (phase current signals) of a wind generator requires increasing attention, particularly by the spectral analysis of their DWT approximations, or details by a PSD technique.

This paper is organized as follows. In Part 2, the mathematical theory of a WT is explained and distinguished for both continuous and discrete versions. In Part 3, the application results of a DWT are illustrated and discussed. Part 4 presents the conclusions. 


\section{WAVELET TRANSFORM THEORY}

A WT has been provided as a solution to eliminate the restrictions in the time-frequency representation existing in other techniques [14]. Essentially, a wavelet is a limited periodical function beginning and ending with the value, zero. Specifically, its function integrates at zero. A WT is simply an expanded FT accompanied by alterable windows. WT applications include compressing of data and images and attenuation of noises.

The mother wavelet, $W(t)$, as suggested by Morlet is expressed as

$$
W_{a, b}(t)=\frac{1}{\sqrt{|a|}} W\left(\frac{t-b}{a}\right) \quad a, b \in R^{+}(a \neq 0)
$$

Where $a$ and $b$ are the dilation and translation parameters that have the role of measuring the scale degree and determining the wavelet time location, respectively. These two parameters assist the process of manipulating the shape of the mother wavelet by scaling and shifting over the signal that is analyzed. The robustness of the wavelets is the capability to adjust simultaneously the resolutions of both the time and frequency [14]. The case where the absolute value of the parameter $a$ is less than 1 corresponds to high frequencies and narrow time widths; otherwise, it is the opposite. This adjustment conforms to Heisenberg's uncertainty principle, which is another time-frequency method.

\subsection{Discrete Wavelet Transform}

Because a continuous WT is considered as a redundant tool in DSP for promoting practical computational applications, it is converted into a numeric form by discretizing the dilation and translation parameters. By analyzing the original signal of $2^{N}$ length in various frequency ranges by using a DWT, each range is related to a specific frequency resolution. By applying low-pass and high-pass filters, the original signal is analyzed to yield approximation and detail coefficients, and the frequency band is halved during each pass. The coefficients of approximation are the values obtained by the low-pass filter, whereas the coefficients of detail are those yielded by the highpass filter. The DWT is obtained by discretizing $a$ and $b$. The important step is the operation of sampling the parameters, $a$ and $b$, to ensure accurate reconstitution of the $i(t)$ resulting from the WT. Depending on the various levels of the discretization process, the WT exists in numerous forms. Assuming that $a=a_{0}{ }^{j}$ and $b=k a_{0}{ }^{j} b_{0}$, where $a_{0}$ $>0, a$ and $b$ can be discretized. Generally, $a_{0}=2$ and $b_{0}=1$; thus, the scale is sampled along a dyadic sequence. By specifically selecting $W(t) \in L^{2}(\Re)$, the following orthogonal wavelet is built by a dyadic discretization:

$$
W_{j, k}(t)=2^{-j / 2} W\left(2^{-j}\left(t-2^{j} k\right)\right)
$$

Because all the function components are orthogonal, this function is also orthogonal, and in this case, the product of all the basic functions yields zero. By the discretization process in equation (2), the reverse DWT of $i(t)$ can be obtained as

$$
i(t)=\sum_{j=-\infty}^{\infty} \sum_{k=-\infty}^{\infty} C_{j, k} W(t)
$$

Where $C_{j, k}$ is defined as the coefficient of the wavelet derived from the original signal, $i(t)$, and dual function, $\bar{W}(t)$, of $W(t)$ as

$$
C_{j, k}=\int_{-\infty}^{\infty} i(t) \bar{W}_{j, k}(t) d t
$$

From the specific approximations and details obtained from the original signal, significant amount of information can be extracted from the DWT. Equation (5) formulates the signal details at each level $j$, whereas equation (6) expresses the signal approximations at level $J$ by collecting all the corresponding details [15-21].

$$
\begin{aligned}
D_{j} & =\sum_{k \in Z} C_{j, k} W_{j, k}(t) \\
A_{J} & =\sum_{k \in Z} D_{j}
\end{aligned}
$$

There is no redundancy because the wavelet that forms the details and approximations through the DWT is lower than the original signal. This remarkable feature is what reduces the computational cost of a DWT compared to that required with a CWT. Thus, equation (7) provides the bilateral composition of the original signal, and equation (8) expresses the reconstructed signal.

$$
\begin{aligned}
& A_{J-1}=A_{J}+D_{J} \\
& i(t)=A_{J}+\sum_{j \leq J} D_{j}
\end{aligned}
$$

Above, $i(t)$ is the real signal, $j$ is the level of decomposition $(j=1,2, \ldots, J)$, and $A_{j}$ denotes the low-frequency coefficients (approximations) and $D_{j}$ denotes the high-frequency coefficients (details) of $y(t)$ at the $j^{\text {th }}$ decomposition level. The decomposition architecture of a $J$-level is illustrated in Figure 1. It is clear that $D_{j}$ and $A_{j}$ are extracted from the high-pass and low-pass filtering at each level with down sampling. Following the decomposition of $y(t)$ by the $J$-level, the filtering process can present the DWT based on the algorithm of Mallat, where the real signal is decomposed into independent frequency bands.

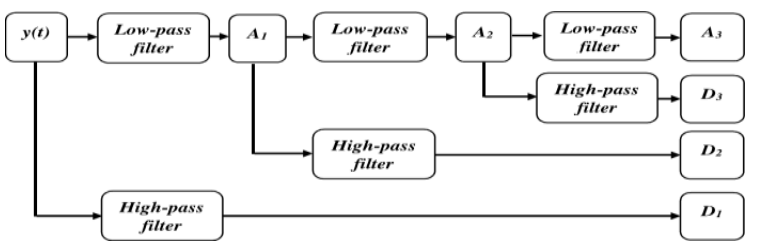

Fig. 1. Example of the decomposition scheme with three levels

Numerous wavelet families, which can be particularly helpful in this study, have been 
included. The following is a list of some basic families: Daubechies, Haar, coiflets, biorthogonal, Morlet, symlets, Meyer, and Mexican hat. In addition, examples of other actual wavelets are the reverse biorthogonal, Gaussian derivatives family, and FIR-based approximation of the Meyer wavelet. The complex wavelet families are the third category including Morlet, Gaussian derivatives, Shannon, and frequency B-spline. All of these wavelets can be used to detect BRB defects. In fact, it is recommended to use a high mother wavelet order to minimize the overlap impact at the cost of a long computation time. However, because of the strength of this applied technique, a low mother wavelet order is also capable of providing reasonable outcomes.

\section{RESULTS OF DWT-PSD APPROACH}

The studied technique is experimentally proved in a PC-based diagnostic system with sampling frequency $f=10 \mathrm{kHz}$ on three squirrel cage induction generators with identical properties and a shaft speed of more than $1500 \mathrm{rpm}$ [22]. The test is conducted for the data obtained from the mentioned three 5.7-kW generators; one with a good health rotor, the second with one BRB, and the third with two BRBs. For each test, the generator is connected to a wind turbine, which acts as a source of mechanical power, and also to an appropriate electric load, as shown in Figure 2.

The objective is to use the power signal density of the phase current generator envelope analyzed by the DWT for the two generators with BRBs (defective) and compared with the first generator (healthy).

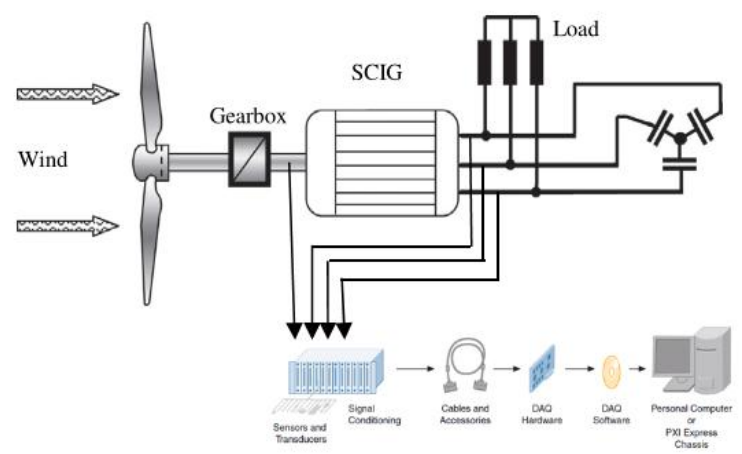

Fig. 2. Renewable Energy System Model

\subsection{Regular PSD results}

When first the sampling stage of the phase current is formed and then analyzed by using a classical method MCSA, such as a PSD technique (precisely, the MUSIC technique), three types of spectra are provided to compare with the PSDs obtained from stator currents $I_{\text {Phase }}$ (traditional technique).

Figure 3 shows the spectra of the stator current in the proximity of the fundamental frequency. When the number of BRBs becomes large, the magnitudes of both the lower and upper components related to the fault are identical. This is also the case for the space between the sideband components and main component. The frequencies related to the states of the BRBs are detectable in the experiments implemented at the load state. For the case of one BRB, the slip is ( $\mathrm{s}=2.5 \%)$, and for the case of two BRBs $(\mathrm{s}=2.6 \%)$. For healthy case ( $s=2.2 \%$ ), the leakage of the spectral density resulting from the main frequency completely eliminates the sideband components.
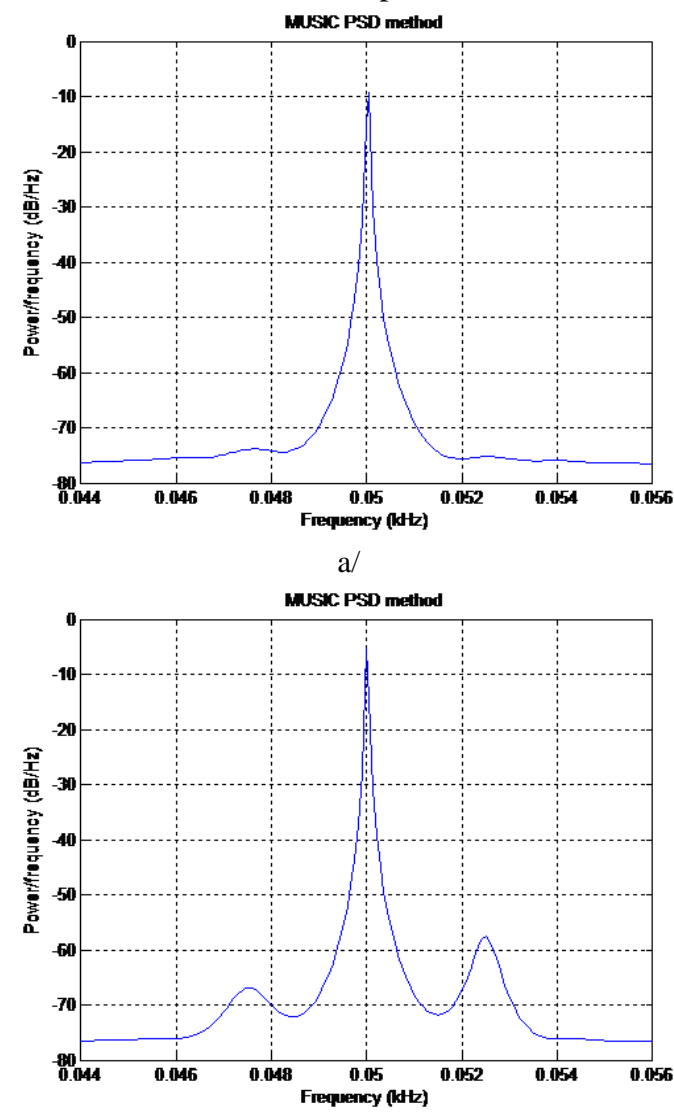

b/

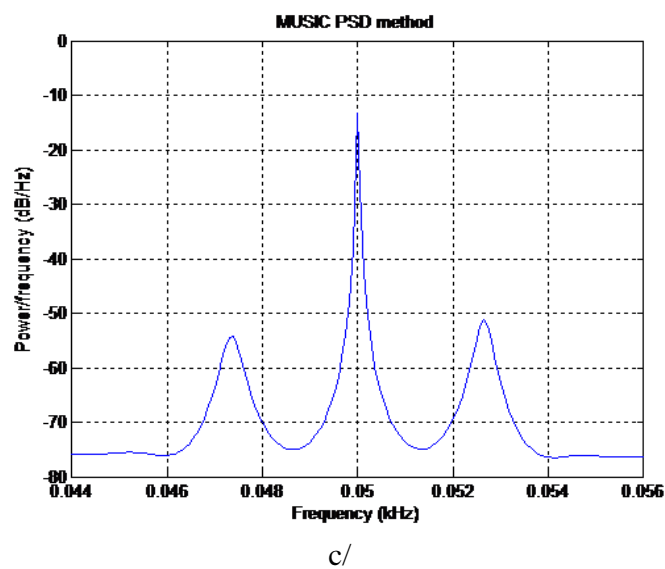

Fig. 3. Regular PSD results, (a) healthy rotor, (b) defective rotor (1 BRB), (c) defective rotor (2 BRBs)

Figure 3 (b) and (c) provide the phase current spectra obtained with one and two BRBs respectively. The lower and upper components associated with the fault, $(1 \pm 2 k s) f_{s}$, are presented clearly along the spectral density axis. 
To confirm that the harmonics, $(1 \pm 2 k s) f_{s}$, shown in the above spectra are induced by the possible existence of BRB faults, the spectrum of the phase current when the squirrel cage induction generator runs with a healthy rotor (figure 3 (a)) is provided and compared with those of the defective rotors. This signal processing contributes to supporting the fact that the presence of a BRB in a generator can create peaks in the spectra at the harmonics, $(1 \pm 2 \mathrm{ks}) f_{s}$.

Note that the spectra peaks illustrated at the harmonics, $(1 \pm 2 \mathrm{ks}) f_{s}$, are certainly produced from the existence of few or numerous broken bars. Therefore, based on this information, it is acceptable to perform the fault diagnosis of rotors by processing the target peaks of the spectra.

For performing fault detection of squirrel cage rotors without having to compare with any reference (reference generated from a healthy generator), the definitive resolution is closely related to the question, "Is the studied rotor defective or not?". This can be done only using a signal that has been analyzed. It is known that all squirrel cage generators have a slight asymmetry due to the construction stage that occurs in the phase current spectra at a low component, $(1-2 k s) f_{s}$. Simultaneously, the speed fluctuation generates an extra component of high frequency $(1+2 k s) f_{s}$ in the phase current spectra, as clearly shown in Figure 3. However, squirrel cage generator manufacturers ensure that the generators offer a slight asymmetry, which can be the major reason of the defects. Therefore, this will be developed in the diagnostic methods. The phase current spectra and particularly the frequency peak at $(1+2 k s) f_{s}$ are discussed. Typically, this peak is extremely weak or equal to zero for a normal generator.

\subsection{DWT-PSD results}

For the PSD estimation of the signal envelope of some DWT approximations and details, the number levels of the DWT approximations and details can be selected. The appropriate number of the decomposition levels $(n)$ relies on the sampling frequency, $f=10 \mathrm{kHz}$, of the generator phase current. A choice must be made for the high-level signals (approximations $A_{j}$ and details $D_{j}$ ) to reach the entire frequency range in which the sideband is located. The level number of the DWT decomposition for obtaining approximation signals $\left(A_{j}\right)$ should be such that the maximum limit of its related frequency band is below the main frequency [23] under the condition:

$$
2^{-(n+1)} f<f_{s}
$$

According to the above-mentioned condition, the level number of the decomposition concerning with the approximation signals, which contain the left and right sideband harmonics, is the integer value, $n$, obtained by the expression

$$
n=\operatorname{int}\left(\frac{\log \left(f / f_{s}\right)}{\log (2)}\right)
$$

This phase current signal should be further decomposed by decomposing the frequency range, $[0-f]$ into more ranges. Typically, it is recommended to add two more levels of decomposition, $(n+2)$, which are suitable for its DWT analysis [23], as

$$
n+2=\operatorname{int}\left(\frac{\log (10000 / 50)}{\log (2)}\right)+2=\operatorname{int}(7.64)+2=9
$$

Where: int is an integer number

Table 1. Multilevel Decomposition with the Corresponding Frequency Bands

\begin{tabular}{|c|c|c|c|c|}
\hline Levels & \multicolumn{2}{|c|}{ Approximations } & \multicolumn{2}{c|}{ Details } \\
\cline { 2 - 5 } & $\mathrm{A}_{\mathrm{j}}$ & $\begin{array}{c}\text { Frequency band } \\
(\mathrm{Hz})\end{array}$ & $\mathrm{D}_{\mathrm{j}}$ & $\begin{array}{c}\text { Frequency band } \\
(\mathrm{Hz})\end{array}$ \\
\hline $\mathrm{j}=1$ & $\mathrm{~A}_{1}$ & $0-5000$ & $\mathrm{D}_{1}$ & $5000-10000$ \\
$\mathrm{j}=2$ & $\mathrm{~A}_{2}$ & $0-2500$ & $\mathrm{D}_{2}$ & $2500-5000$ \\
$\mathrm{j}=3$ & $\mathrm{~A}_{3}$ & $0-1250$ & $\mathrm{D}_{3}$ & $1250-2500$ \\
$\mathrm{j}=4$ & $\mathrm{~A}_{4}$ & $0-625$ & $\mathrm{D}_{4}$ & $625-1250$ \\
$\mathrm{j}=5$ & $\mathrm{~A}_{5}$ & $0-312.5$ & $\mathrm{D}_{5}$ & $312.5-625$ \\
$\mathrm{j}=6$ & $\mathrm{~A}_{6}$ & $0-156.25$ & $\mathrm{D}_{6}$ & $156.25-312.5$ \\
$\mathrm{j}=7$ & $\mathrm{~A}_{7}$ & $0-78.12$ & $\mathrm{D}_{7}$ & $78.12-156.25$ \\
$\mathrm{j}=8$ & $\mathrm{~A}_{8}$ & $0-39.06$ & $\mathrm{D}_{8}$ & $39.06-78.12$ \\
$\mathrm{~J}=9$ & $\mathrm{~A}_{9}$ & $0-19.53$ & $\mathrm{D}_{9}$ & $19.53-39.06$ \\
\hline
\end{tabular}

The DWT decomposition tree is given in Table 1. The frequency ranges associated with the broken BRB defects for each level are clearly provided in this table. Therefore, the decomposition levels that are extremely important for our study are from $A_{I}$ until $A_{7}$ concerning the approximations and $D_{8}$ for the details. From these approximations and the single detail, we selected two approximations at level 6 with a frequency range of [0-156.25] and at level 7 with a frequency range of [0-78.12]. Moreover, the selected single detail at level 8 with a frequency range of [39.06-78.12], as clearly presented in Figure 4, is ensured continuously for the three cases of the studied generators. This selection was made to avoid the high-frequency components that exist in other approximation frequency ranges and are not necessary for BRBrelated faults. Following the PSD estimation analysis step of approximations $A_{6}$ and $A_{7}$ and detail $D_{8}$, it became clear that this analysis of cannot provide good and encouraging results to compare them with those derived from a regular signal for the BRB characteristic defects, $f_{s} \pm 2 s f_{s}$. This is despite there being numerous data samples from both the vectors being processed. Therefore, it is logical to use the only and remaining approximation at level 6, which provides the best results, for comparison with the results obtained from the original phase current signal. 

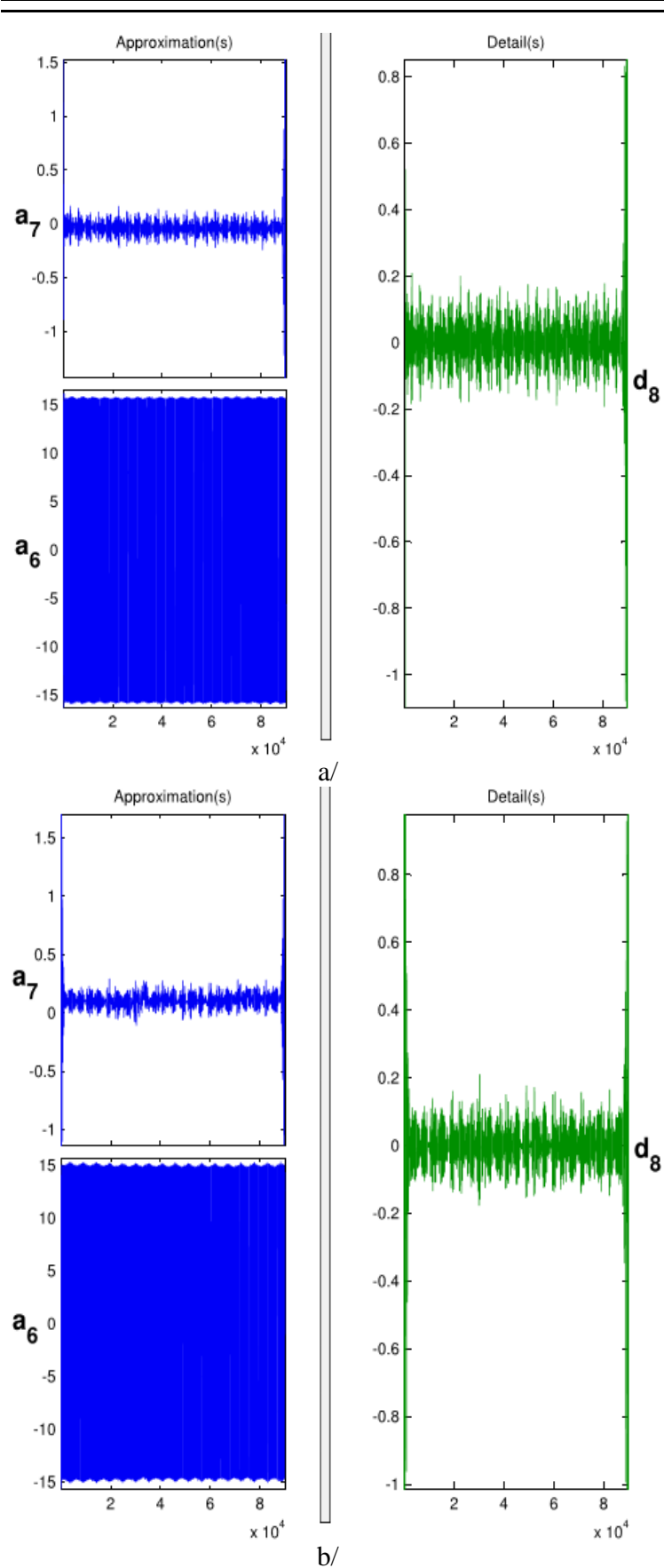

To validate the proposed method, Table 2 lists the generator conditions, measured speeds, slip percentages, and frequencies of the theoretical sideband components related to the studied fault for the regular and transformed signals.

The above-mentioned current data were transformed with the Daubechies-44 mother wavelet using the wavelet toolbox of MATLAB software, and the PSD (exactly the MUSIC method) was used to analyze them. Their spectra in the three cases are presented in Table 2 and shown again in Figure 5 based on the DWT method with the approximation at level 6 . We select this from all the approximations and details because its best frequency band between 0.0 and $156.25 \mathrm{~Hz}$ contains only the fundamental frequency, $f_{s}$, and its sideband frequencies, $f_{s} \pm 2 s f_{s}$.
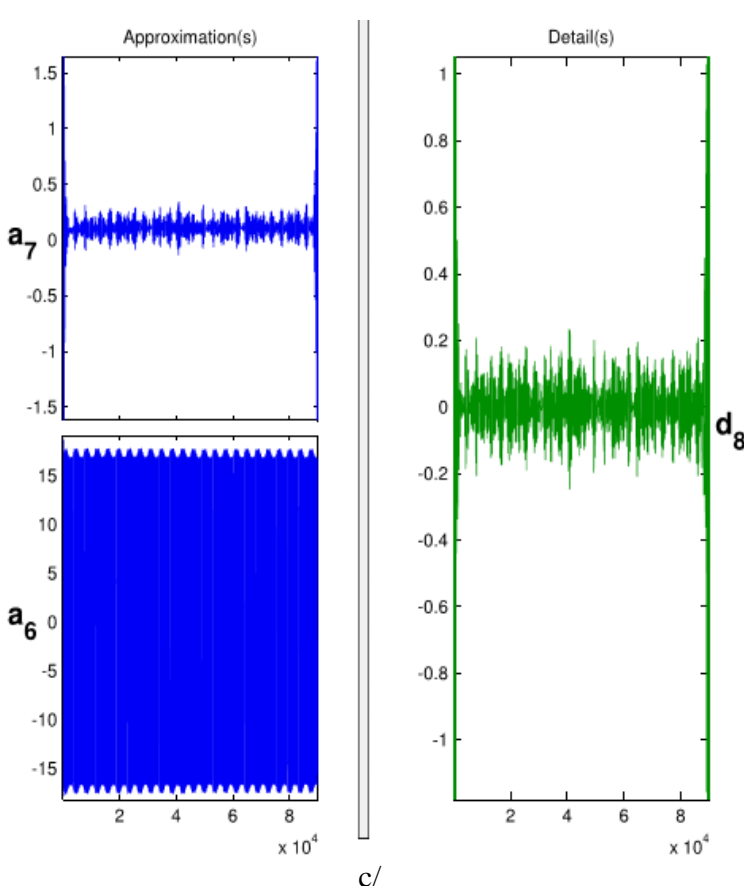

Fig. 4. Selected approximations and detail for (a) healthy rotor, (b) defective rotor (1 BRB), (c) defective rotor (2 BRBs)

Table 2. Generators Conditions with Sideband Components from the Regular and DWT Signals

\begin{tabular}{|c|c|c|c|c|}
\hline $\begin{array}{c}\text { Generator } \\
\text { condition }\end{array}$ & $\begin{array}{c}\text { Speed } \\
(\mathrm{rpm})\end{array}$ & $\begin{array}{c}\text { Slip } \\
(\%)\end{array}$ & $\begin{array}{c}\text { Regular signal } \\
f_{s} \pm 2 s f_{s}(\mathrm{~Hz})\end{array}$ & $\begin{array}{c}\text { DWT }\left(\mathrm{A}_{6}\right) \\
f_{s} \pm 2 s f_{s}(\mathrm{~Hz})\end{array}$ \\
\hline Healthy & 1533 & 2.2 & $-73.86-75.24$ & $-57.62-55.49$ \\
$(1 \mathrm{BRB})$ & 1538 & 2.5 & $-66.84-57.57$ & $-46.64-50.00$ \\
$(2 \mathrm{BRB})$ & 1539 & 2.6 & $-54.14-51.25$ & $-39.60-40.17$ \\
\hline
\end{tabular}

The PSD estimation results of the approximation at level 6 for the three cases of generators: healthy rotor, rotor with one BRB, and rotor with two BRBs, are presented clearly in Figure 5 (a), (b), and (c) consecutively.

The outcomes derived from the regular signal, as shown in Figure 3, and those obtained from the approximation at level 6, as shown in Figure 5, can be compared. Next, by using the peak values of the sideband components for the two cases provided in Table 2, the following points of interest may be drawn:

- From the graphs of both the methods, it is noticed that the frequencies associated with the BRBs are the same as those for the regular phase current signal and DWT approximation at level 6 in each studied case of the three generators.

- Based on both the graphs and Table 2, the peaks of the sideband components, $f_{s} \pm 2 s f_{s}$, significantly increase. For example, the defective generator with 2 BRBs has peaks of [$54.14 \mathrm{~dB}-51.25 \mathrm{~dB}$ ] for the regular signal and [$39.60 \mathrm{~dB}-40.17 \mathrm{~dB}]$ for the DWT approximation at level 6, i.e., the differences are [14.54 dB $11.08 \mathrm{~dB}$ ]. The defective generator with $1 \mathrm{BRB}$ has peaks of [-66.84 $\mathrm{dB}-57.57 \mathrm{~dB}$ ] for the regular signal and $[-46.64 \mathrm{~dB}-50.00 \mathrm{~dB}]$ for the DWT approximation at level 6 . 
Therefore, the differences are [20.20 dB 7.57 $\mathrm{dB}]$, which are large values in terms of the PSD.

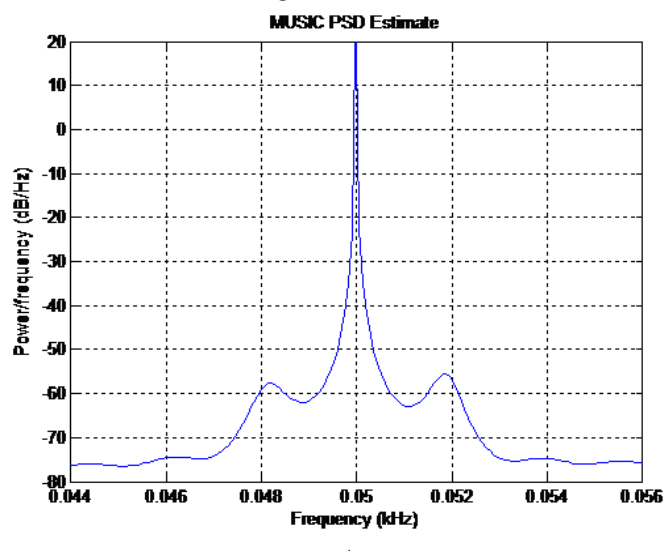

a/

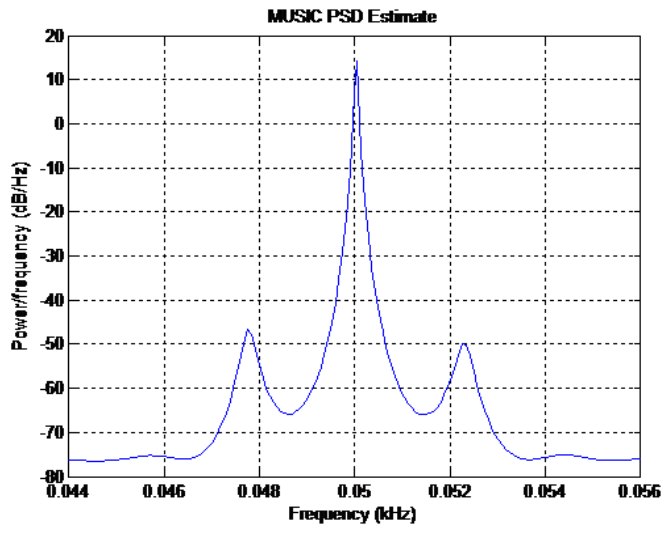

$\mathrm{b} /$

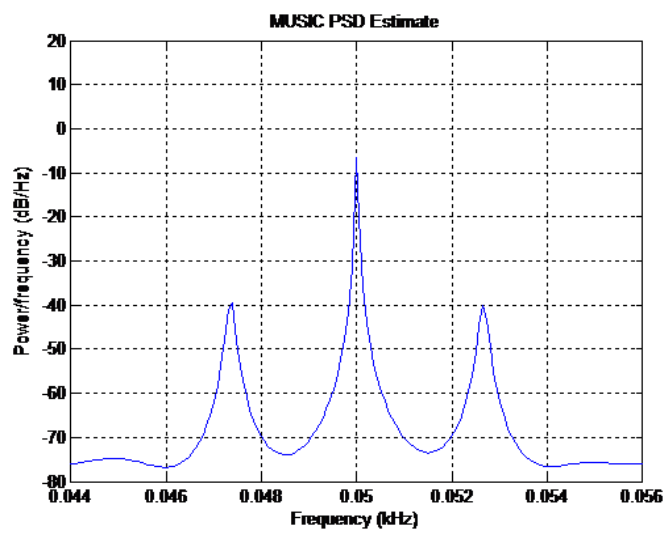

c/

Fig. 5. DWT-PSD results of the approximation at level 6 (a) healthy rotor, (b) defective rotor (1 BRB), (c) defective rotor (2 BRBs)

- The information provided by the DWT approximation at level 6 is clearly important, particularly in terms of the spectral density, and it can complement that offered by the regular phase current to achieve more accurate detection. This scenario is an example of a health policy if the PSD method is not sufficient to be employed.

\subsection{Discussions}

The number of levels required for the studied technique is calculated from the sampling frequency of the data acquisition system and fundamental frequency of the generator. Two additional levels are added for more decomposition of the approximations and details necessary for the DWT analysis.

The frequency bands of both the approximations and details for each of the nine levels are identified. Subsequently, they are neutralized to exclude the characteristic BRB frequencies, which remain in the required frequency bands.

Among the selected frequency bands, those with high frequencies are abandoned to avoid spectral interference. Therefore, for the study and analysis, there are only three frequency bands, and they are related to $A_{6}, A_{7}$, and $D_{8}$. Following the digital processing phase, it is found that only the $A_{6}$ frequency band is capable of delivering excellent results.

The PSD is applied to transform the stator current vector into a representation in the frequency domain. It may be the most robust estimation and one of the main tools utilized in DSP.

A DWT is applied to set the stator current signature for generating increasing information by producing an approximation and detail decomposition of that signature. The DSP utilized for the fault diagnosis is established based on the analysis of the regular phase current signal and its approximation at level 6 to determine their difference. This is done because this difference is extremely important in cases of low slips and certain to occur in cases of low loads. The DWT serves like a brave knight on the chess board of DSP, provided our generosity is immense.

The DWT studied above is extremely nonlocal, and complete samples of the phase current are necessary to generate the approximations and details decomposition at each sampling point. Producing the approximations and details decomposition of a sampled phase current requires low-pass and high-pass filters. The results obtained from this method, particularly the peaks associated with the sideband components, motivates its combination with some artificial intelligence methods, such as a fuzzy logic system, to identify the type as well as to count the BRB defects, while being suitable for online implementations.

\section{CONCLUSION}

In this work, a DWT based on the PSD is offered for diagnosing the presence of BRB defects in a squirrel cage induction generator by using the stator current. Therefore, a comparison study of two methods for BRB fault detection is performed, proved, and improved. First, a method uses the estimated PSD and allows traditional detection of BRBs by applying the power spectrum analysis to the phase current of the squirrel cage induction generator operating under the stationary condition. The fault detection is accomplished by analyzing both the sideband components (lower and upper) on both the sides of the main frequency component. 
This traditional method has important features such as simplicity of the DAQ systems and needed software, robustness, and excellent outcomes, at least until now. However, under several conditions, such as insufficiently non-loaded generators where the slip $(s)$ is extremely small and both the sideband components nearly overlap with the main frequency, they are difficult to observe and therefore difficult to use for diagnosis. The second method uses a DWT. Therefore, this allows the decomposition of the phase current into numerous levels of frequency bands, which provides us the opportunity to study any approximation or detail individually in its frequency band. The benefit of a DWT over a decomposition step is the analysis of the information included in an unsteady signal at various time-frequency representations. The DWTPSD approach may be considered as an extension of the PSD obtained from the original signal under unsteady conditions. Finally, this type of technique can represent a new trend to achieve fault diagnosis under several conditions where the PSD obtained from the original signal is not efficient.

\section{REFERENCES}

1. Pons Llinares J, Climente Alarcón V, Vedreño Santos F, Antonino Daviu J, RieraGuasp M. Electric machines diagnosis techniques via transient current analysis. Proceedings of the 38th Annual Conference of the IEEE Industrial Electronics Society, IECON 2012, 25-28 October, Montreal, Canada.

2. Kia SH, Henao H, Capolino GA. Efficient digital signal processing techniques for induction machine fault diagnosis. Proc. IEEE Workshop Electrical Machines Design, Control and Diagnosis, WEMDCD 2013, Mar. 11-12, Paris, France.

3. Thomson WT, Fenger M. Current signature analysis to detect induction motor faults. IEEE Industry Applications Magazine 2001;26-34.

4. Cai H, Sun Q, Wood D. Condition monitoring and fault diagnosis of a small permanent magnet generator. Wind engineering. 2016;40(3), 270-282. https://doi.org/10.1177/0309524X16647842

5. Castellani F, Astolfi D, Becchetti M. Berno F. Experimental damage detection on small wind turbines through vibration and acoustic analysis. Proceedings of ISMA 2018 - International Conference on Noise and Vibration Engineering and USD 2018 - International Conference on Uncertainty in Structural Dynamics, 2018:4793-4807.

6. Jinglong Chen, Jun Pan, Zipeng Li, Yanyang Zi, Xuefeng Chen. Generator bearing fault diagnosis for wind turbine via empirical wavelet transform using measured vibration signals. Renewable Energy. 2016;89:80-92. https://doi.org/10.1016/j.renene.2015.12.010

7. Wei Teng, Xian Ding, Hao Cheng, Chen Han, Yibing Liu, Haihua Mu. Compound fault diagnosis and analysis for a wind turbine gearbox via a novel vibration model and empirical wavelet transform. Renewable Energy, 2019;136:393-402. https://doi.org/10.1016/j.renene.2018.12.094

8. Jianming Ding, Chengcheng Ding. Automatic detection of a wheelset bearing fault using a multilevel empirical wavelet transform. Measurement.
2019:134: 179-192.

https://doi.org/10.1016/j.measurement.2018.10.064

9. Dong Wang, Kwok-Leung Tsui, Yong Qin. Optimization of segmentation fragments in empirical wavelet transform and its applications to extracting industrial bearing fault features. Measurement, 2019;133:328-340. https://doi.org/10.1016/j.measurement.2018.10.018

10. Baojia Chen, Baoming Shen, Fafa Chen, Hongliang Tian, Wenrong Xiao, Fajun Zhang, Chunhua Zhao. fault diagnosis method based on integration of RSSD and wavelet transform to rolling bearing. Measurement. 2019;131:400-411. https://doi.org/10.1016/j.measurement.2018.07.043

11. Jaskaran Singh, Darpe AK, Singh SP. Rolling element bearing fault diagnosis based on OverComplete rational dilation wavelet transform and auto-correlation of analytic energy operator. Mechanical Systems and Signal Processing. 2018;100:662-693. https://doi.org/10.1016/j.ymssp.2017.06.040

12. Ping Ma, Hongli Zhang, Wenhui Fan, Cong Wang. Early fault diagnosis of bearing based on frequency band extraction and improved tunable Q-factor wavelet transform. Measurement, In press, accepted manuscript, 2019;1137: 189-202 https://doi.org/10.1016/j.measurement.2019.01.036

13. Xin Zhang, Zhiwen Liu, Jiaxu Wang, Jinglin Wang. Time-frequency analysis for bearing fault diagnosis using multiple Q-factor Gabor wavelets. ISA Transactions. 2019;87:225-234. https://doi.org/10.1016/j.isatra.2018.11.033

14. Yan R, Gao RX, Chen X. Wavelets for fault diagnosis of rotary machines: a review with applications. Signal Processing 2014; 96:1-15.

15. Antonino Daviu JA, Riera Guasp M, Pineda Sánchez M, Pérez R B. A critical comparison between DWT and Hilbert-Huang-Based methods for the diagnosis of rotor bar failures in induction machines. IEEE Transactions on Industry Applications 2009;45:17941803.

16. Daubechies I. Ten lectures on wavelets. SIAM, Philadelphia, PA. 1992.

17. Mallat S. A wavelet tour of signal processing. 2nd. Edition, Academic Press, San Diego, California, USA. 1999.

18. Strang G, Nyugen T. Wavelets and Filter Banks, Wellesley Cambridge Press, Wellesley, MA, USA, 1996.

19. Qian, S. Introduction to Time-Frequency and Wavelet Transforms, Prentice Hall PTR, 2002.

20. Mallat SG. A theory for multiresolution signal decomposition: the wavelet representation. IEEE Transactions on Pattern Analysis and Machine Intelligence. 1989;11(7): 674-693.

21. Rafiee J, Rafiee MA, Tse PW. Application of mother wavelet functions for automatic gear and bearing fault diagnosis. Expert Systems with Applications, 2010; 37(6): 4568-4579.

22. Lahcène Noureddine, Touhami O. Diagnosis of wind energy system faults Part I : Modeling of the squirrel cage induction generator. International Journal of Advanced Computer Science and Application (IJACSA) 2015;6:46-53.

23. Daviu JA, Riera Guasp M, Roger Folch J, Martínez Giménez F, Peris A. Application and optimization of the discrete wavelet transform for the detection of 
broken rotor bars in induction machines. Appl. Comput. Harmon. Anal. 2006;21:268-279.

Received 2019-04-09

Accepted 2019-07-03

Available online 2019-07-04

Dr. Lahcène Noureddine

was born in Aflou, Algeria in

1980, He received the State

Engineer degree in Electrical

Engineering in 2002 on

electrical machines from

Laghouat University, the

Magister degree on electrical

machines in 2005 from

National Polytechnic School

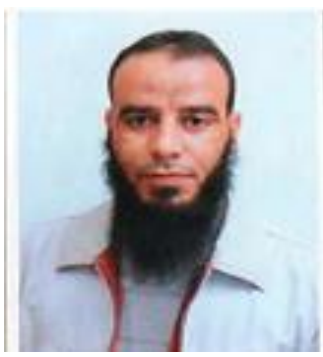

of Algiers, the $\mathrm{PhD}$ on industrial diagnostics in 2019 from the University of Djelfa. He is an assistant professor of Electrical Engineering at Laghouat University. His research interests are modelling, parameter estimation, and fault detection of electrical machines.

\section{Prof. Ahmed HAFAIFA was}

born in Algeria in 1974, he

received the State Engineer

degree in 2000 on Applied

Automation, the Magister

degree in 2004 on Applied

Automation and control systems and the $\mathrm{PhD}$ on

Applied Automation and Signal Proc the UMBB Boumerdes University. He received the Habilitation from the University of Sciences and Technology Houari Boumediene - USTHB - Faculty of Electronics and Computer Science, Department of Instrumentation and Automation on 2012. He is a $\mathrm{PhD}$ and Full Professor in Industrial Process: Automation / Diagnosis and Reliability Engineering at the Science and Technology Faculty of the University of Djelfa, Algeria, where $\mathrm{He}$ is actually the president of the scientific committee of Technical Sciences Department (CSD) since 2014 and he is pursuing his researches as a researcher at the Applied Automation and Industrial Diagnostic Laboratory of the University of Djelfa. Professor Ahmed HAFAIFA has participated in several international research projects and has led several national research projects. Currently he is the Director of the Applied Automation and Industrial Diagnostic Laboratory of the University of Djelfa.

Prof. Kouzou Abdellah (IEEE Senior memebr \& IACSIT Senior member, IFAC,IAENG \& IISRO member, IEEE-HKN Alumni Member) was born in Djelfa, Algeria in 1964. $\mathrm{He}$ received the State Engineer degree, the Magister, the $\mathrm{PhD}$, and the

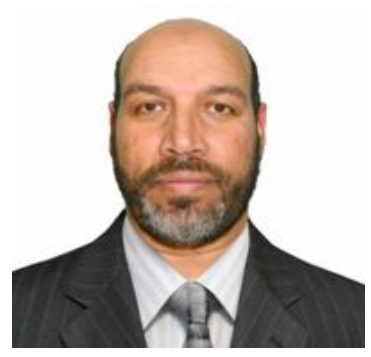

Habilitation from University of Tiaret, Algeria, University of Boumerdes, and Polytechnics Superior National School respectively. He has been a researcher with Technische Universität of Muenchen in Germany form 2010, 2011 and 2012. He is a collaborator collaborator researcher at Texas A\&M University at Qatar. He is an associate professor with the faculty of
Sciences and Technology at Djelfa university in Algeria, where he was the president of the Scientific council of the faculty since June 2014. Dr. Kouzou has participated in several research projects and has led several research projects. Currently he is the Dean of the faculty of Sciences and Technology at Djelfa university in Algeria. 